\title{
Uveitis: Pathogenesis, Clinical presentations and Treatment
}

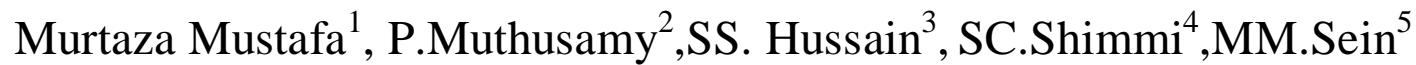 \\ ${ }^{1,3,4,5,}$ Faculty of Medicine and Health Sciences, University of Malaysia, Sabah, Kota Kinabalu, Sabah, Malaysia \\ 2. Kota Kinabalu Specialist Centre, Kota Kinabalu, Sabah, Malaysia
}

\begin{abstract}
Uveitis a vision threatening inflammation of uvea is prevalent worldwide. In the United States uveitis has prevalence of 2.3 million people and causes $10 \%$ of all cases of blindness. Uveitis is classified by the ocular structures involved e.g., anterior, intermediate, posterior and panuveitis.0cular TB(Eales' disease) is common in patients from Indian sub-continent. Frequent uveitis etiologies includes, Herpes simplex, Herpes zoster, cytomegalovirus, syphilis, tuberculosis, Lyme disease, toxoplasmosis and fungi. Diagnosis of uveitis is always presumptive and cannot be proved by pathology or by culture. PCR has proven most useful in diagnosis of uveitis. Treatment is mostly by acyclovir, intravenous antiviral agents, anti TB drugs for ocular tuberculosis, intravenous penicillin for ocular syphilis and sulfadiazine for Toxoplasmosis, with corticosteroids added to prevent intraocular or sight threatening inflammation. Early diagnosis and treatment of uveitis can prevent vision loss.
\end{abstract}

KEYWORS: Uveitis, Acute retinal necrosis, Progressive outer retinal necrosis, Herpetic anterior uveitis, and Treatment.

\section{INTRODUCTION}

Uveitis the inflammation of uvea (Latin word uva,meaning grape).Uveitis is classified by the ocular structures involved, International Uveitis Study Group classification (i.e., anterior, intermediate, posterior , and panuveitis) is commonly used[1].In anterior uveitis. inflammation involves the iris(iritis),anterior ciliary body(cyclitis),or both (iridocyclitis) Posterior uveitis refers to inflammation involving the choroid(choroditis),retina (retinitis), both( chororetinitis), or retinal vessels(retinal vasculitis)Panuveitis involves all three parts of the uvea .Uveitis may extend to involve the cornea(keratouveitis) or sclera (sclerouveitis)[1].Uveitis is prevalent worldwide. In the United States uveitis has prevalence of 2.3 million people and causes $10 \%$ of all cases of blindness[2].The prevalence in the United States is 70 to 115 cases per 100,000 population, and uveitis affects slightly more woman than men[3].The prevalence in developing countries is not clear, but a study from West Africa found that uveitis including that due to onchrocerciasis caused $24 \%$ of blindness[4].Recurrent retinal and vitreous hemorrhages in ocular TB(Eales' disease) is most common in patients from India, Pakistan and Afghanistan[5].Uveitis can occur at any age, but the average age at presentation is about 40 .Anterior uveitis is the most common type of uveitis, accounting for the $90 \%$ of uveitis cases in community based ophthalmology practice and approximately 50\% in university referral centers [6]. Intermediate uveitis, posterior and panuveitis each account for 10 to $15 \%$ of uveitis cases in university referral centers but only $1 \%$ to $5 \%$ in community practices[6].Most common uveitis infectious agents include: in anterior uveitis, herpes simplex, Herpes zoster, syphilis, tuberculosis, in intermediate uveitis, Lyme disease and in posterior uveitis, toxoplasmosis, tuberculosis, fungi(candida),Herpes simplex, zoster(ARN),cytomegalovirus, and in panuveitis, syphilis ,tuberculosis and leptospirosis[7]. Treatment of uveitis is based upon clinical presentation and infectious agent. Treatment with corticosteroids is effective in controlling inflammation and improving vision, but in patients with immune recovery uveitis (IRV) may require surgery [8].The paper reviews the pathophysiology, clinical presentations and treatment of uveitis.

\section{INFECTIOUS AGENTS AND PATHOPHYSIOLOGY}

Uveitis may be caused by autoimmune conditions, infections, or rarely, trauma, but $50 \%$ of cases are idiopathic. Some cases of intraocular inflammation masquerade as uveitis (masquerade syndromes) but other causes such as malignancy (e.g. ocular-central nervous system lymphoma).Infectious uveitis nearly always results from hematogenous spread of infection from another part of the body to the highly vascular uvea [1].The pathophysiology of uveitis depends on the specific etiology but in all types there is breach in the blood-eye barrier. The blood-eye barrier, similar to the blood -brain barrier normally prevents the cells and large protein entering the eye. Inflammation causes this barrier to break down, and WBCs enter the eye .Neutrophils predominate in acute uveitis cases, and mononuclear cells predominate in chronic cases [1].

Infections cause about $20 \%$ of all uveitis cases, with the most common infectious etiologies being herpetic infections and toxoplasmosis [6].The likelihood of an infectious etiology, however, is greater in some categories of uveitis, such as posterior uveitis than others. Understanding the types of infectious etiologies by category is 
helpful in considering possible etiologies for a patient with uveitis. In anterior uveitis, most cases are idiopathic $(40 \%)$ or associated with rheumatologic conditions $(45 \%)$, such as seronegative arthropathies, HL A-B27associated disease.Reiter's syndrome and juvenile rheumatoid arthritis. The most common infectious cause is herpetic anterior uveitis which accounts for $10 \%$ of cases, whereas syphilis, TB, and Lyme disease cause less than $1 \%$.Intermediate uveitis most often has an unknown etiology(69\%) or due to sarcoidos is (22\%) or multiple sclerosis $(8 \%)$;infections are extremely rare[9].Posterior uveitis has an infectious etiology in more than $40 \%$ of cases.Toxoplasmosis causes $25 \%$ to $40 \%$ of cases, whereas less common infectious etiology include cytomegalovirus(CMV) retinitis, acute retinal necrosis(ARN), Toxocara, syphilis, and Candida[9].Major noninfectious diagnoses in a U.S. study were lupus erythematosus, sarcoidosis, and birdshot retinochoroidopathy, an eye disease of unknown etiology( $8 \%$ each)[9].In panuveitis, infections cause of $10 \%$ cases and include syphilis,TB, and Candida[9].The remaining $90 \%$ of cases are caused by sarcoidosis. Behcet's disease, systemic lupus erythematosus, multifocal choroiditis, and panuveitis, or are idiopathic [1].

In Immune recovery uveitis(IRU) although pathogenesis is not entirely certain, it appears to represent an inflammatory reaction to either cytomegalovirus antigen in the eye or low or subclinical levels of cytomegalovirus replication and this inflammatory reaction occurs as the immune system recovers competency[10].Speculation on the pathophysiology of IRU includes the fact that the intraocular inflammation is a reaction to antigenically altered retinal or glial cells adjacent to the healed CMV lesions or secondary to subclinical viral replication along the border of healed CMV[11]. Modorati et al., suggested that all patients with clinical and ophthalmological characteristics of IRU showed the presence of HLA B 8-18[12].

\section{GENERAL CLINICAL CHARACTERISTICS}

Patients usually present with a painful red eye and decreased vision. There may be pupillary constriction, photophobia, and tearing. On slit-lamp examination, there are cells and "flare" (protein) in the anterior chamber. The inner surface of the cornea may be speckled with keratic precipitates that either fine ("granular") or globular ("granulomatous" or "mutton fat" keratic precipitates).The term "granulomatous" is descriptive and does not refer to finding granulomas on pathology. Granulomatous keratic precipitates are less common than granular precipitates and more often associated with sarcoidosis, syphilis, or tuberculosis. Typical steroids may change granulomatous keratic precipitates to granular keratic precipitate [13].

\section{CLINICAL PRESENTATIONS AND ETIOLOGY}

The most common infectious etiologies of uveitis include herpes simplex virus(HSV), varicella zoster virus(VZV),cytomegalovirus(CMV), syphilis, TB, Toxoplasma, and Toxocara.In the United States, other etiologies include Lyme- related uveitis, and chronic endophthlamitis due to Propinobacterium acnes or Candida that mimic uveitis[1].

HVS-Herpetic Anterior Uveitis, of the $10 \%$ of anterior cases that are due to infection, nearly all are caused by HVS .Most patients with HVS-related anterior uveitis either have a history of HVS keratitis or have active corneal infection at the time of uveitis .HVS is common, and approximately $40 \%$ of patients with ocular herpetic disease have recurrent episodes of anterior uveitis[14].Iris in an eye with previous herpetic keratitis should be considered herepetic until proved otherwise[15].Herpetic anterior uveitis is nearly always unilateral. Patients complain of eye pain, redness, and photophobia. The cornea may appear cloudy and slit-lamp examination may reveal interstitial keratitis typical of active recurrent HVS keratitis or corneal scars from prior episodes. Anterior chamber inflammation may be mild to severe, and there may be a hypopyon or keratitis precipitates, or both; keratitic precipitates may be small, large, or stellate. Anterior uveitis due to HVS may occur in a patient with no history of or finding suggesting herpetic keratitis, but this is thought to be uncommon [16].In the absence of clinical evidence or history of HVS keratitis, there are some clues that suggest herpetic anterior uveitis. These include unilateral disease, decreased corneal sensation, posterior synechiae, acute increase in intraocular pressure (from inflammation of the trabecular meshwork), and iris atrophy (either patchy, sectoral,or diffuse )[17].Sectoral iris atrophy is particularly suggestive of HVS or VZV as the etiology [18]. Recent polymerase chain reaction (PCR) studies of the aqueous in patients with no history of keratitis but recent episodes of anterior uveitis with sectoral iris atrophy found HVS in $83 \%$ and VZV in $13 \%$ of patients [19].

Acute Retinal Necrosis (ARN).ARN was first described in 1971 and is a rapidly progressive necrotizing retinitis due to the herpes viruses that mainly affects immunocompetent patients[20].The American Uveitis Society has established the four diagnostic criteria for ARN:(1)focal well demarcated areas of retinal necrosis located in the peripheral retina.(2)rapid circumferential progression of necrosis;(3) occlusive vasculopathy; and (4) prominent inflammation(white blood cells) in the vitreous and aqueous[21].Herpes simples virus type 1 and type 2 and VZV cause nearly all cases. A study on 18 ARN patients(negative for immunodeficiency virus[HIV]) who underwent vitreous biopsy found PCR evidence of VZV in 12,HVS in 4;2 were negative by PCR[22].CMV is a rare cause of ARN but must be considered in immunocompromised patients. Some patients with ARN due to HVS have a history of congenital herpes or herpes encephalitis months to years earlier, but these are uncommon 
[23].ARN typically begins with a unilateral anterior uveitis. Patients may have mild eye pain or photophobia, then decreased vision in the affected eye. Funduscopic examination with an indirect ophthalmoscope shows one or more foci of retinal necrosis in the peripheral retina, an area not usually seen with a direct ophthalmoscope. The areas of retinitis have sharply demarcated borders and typically spread circumferentially and posteriorly. Vascular sheathing develops along with a dense vitritis.Necrosis of areas of the retina leads to retinal detachment in many patients. Retinal detachment occurs weeks to months after onset of ARN.One study found that $50 \%$ of patients developed a retinal detachment, and this occurred 3 weeks to 5 months of ARN[22].Treatment with intravenous acyclovir halts progression of the retinitis in ARN in most cases[24]

Outer Retinal Necrosis or progressive retinal necrosis .PORN is very rare in the United States, but it is a herpetic retinitis that shares many features with ARN.Unlike ARN, however PORN is never seen in immune competent patients, and almost all cases have occurred in HIV-positive patients. Patients with HIV and PORN typically have CD4 counts less than 100mm( average 20)[2].A few cases of PORN have been described in other immunocompromised patients, such as organ transplant patients[25].Nearly all cases of PORN are due to VZV although CMV and HVS have been described. Patients present with vision loss that may be unilateral or bilateral and examination shows multiple peripheral lesions in the deep (outer) layers of the retina initially. These lesions rapidly coalesce to involve the full thickness of the retina. PORN resembles ARN but is distinguished from it clinically by the following three features:(1) involvement of outer retina ;(2) the absence of any inflammation in the vitreous or aqueous humor; and (3) the absence of involvement of the retinal vasculature.0ptic neuropathy may precede PORN in rare cases [26].The retinitis in PORN usually progress rapidly despite systemic antiviral therapy, although recent successes have been achieved by a combination of systemic and intravitreal therapy [1].

Cytomegalovirus Retinitis .CMV may cause ARN in an immunocompromised host, but more typically cause the characteristic CMV retinitis. CMV retinitis affected more than $30 \%$ patient with acquired immunodeficiency syndrome (AIDS) in the pre-highly active antiretroviral therapy (HAART) era, but now rare in countries such as United States, where HAART is widely used [1].CMV retinitis is still seen commonly in patients who do not have good access to antiretroviral medications, however, patients with CMV have painless loss of vision. Eye findings typically include fluffy white retinal infiltrates; retinal vasculitis, which may have frosted branch angiitis pattern; and multiple retinal hemorrhages. An important clinical feature is the absence of significant vitreous inflammation. As a consequence, the view of the retina is clear; that is in contrast to ocular toxoplasmosis, in which vitritis is common [1].

Ruiz-Cruz et al., reviewed charts of 75 patients with CMV retinitis on HAART initiation or during 6 subsequent months, 20 patients had improvement of CMV retinitis. The remaining 55 patients experienced CMV-IRR(cytomegalovirus Immune recovery retinitis);35 of those developed CMV-IRR after HAART initiation(unmasking CMV-IRR);20 experienced paradoxical clinical worsening of retinitis(paradoxical CMVIRR).Nineteen patients with CMV-IRR had $\geq 50 \mathrm{CD} 4 \mathrm{~T}$ cells $/ \mathrm{mm}^{3}$.Six patients with CMV-IRR subsequently developed immune recovery uveitis(IRU).The authors propose definition for CMV-IRR as the condition which is likely to occur after successful ignition of HAART , even in patients with high CD4 T cell count [8].

Syphilis .Ocular syphilis may be the presenting feature of syphilis ,especially in the older adults[27].Syphilis may involve the cornea as an interstitial keratitis or the sclera as a nodular scleritis [28].Uveitis is the most common manifestation of ocular syphilis and is often granulomatous. Syphilis may produce anterior uveitis, intermediate uveitis ,posterior or panuveitis. Syphilitic anterior uveitis is granulomatous in two thirds of patients and bilateral in half [29].Retinal vasculitis may occur, and branch retinal vein occlusions have been described[30].Uveitis may occur in either congenital or acquired syphilis. Typical findings in congenital syphilis disease include interstitistial keratitis and so-called salt-and- pepper fundi. Interstitial keratitis does not usually occur until the patient is teenager or young adult. It may be accompanied by an anterior uveitis. Glaucoma may result from the inflammation. In acquired syphilis, onset of uveitis may occur in secondary or tertiary syphilis. The most common ocular finding in secondary syphilis is iritis, which accounts for more than $70 \%$ of eye findings [30].The diagnosis may be missed if only rapid plasma regain(RPR) or Venereal Disease Research Laboratory(VDRL) is checked because these tests are often negative in tertiary syphilis. In a series of 50 patients with a reactive absorbed fluorescent treponemal antibody (FTA-abs) and eye findings consistent with active or inactive ocular syphilis (e.g.,chorioretinitis ,optic atrophy, iritis interstitial keratitis), the average age was 59,and VDRL was reactive in only $24 \%$ [31].False tests may results may also occur in patients with uveitis, especially as many have underlying rheumatic conditions that increase the risk of a false test[1].

Tuberculosis.0cular complications of TB are rare. Ocular TB was diagnosed in only 1.4\% of the 10,524 patient seen in the eye clinic of Boston sanatorium between 1940 and 1966[32].A 1996 report of 1005 patients in India with active $\mathrm{TB}$ also found evidence of ocular disease in only 1.4\%[33].Ocular disease may be more common in HIV-positive patients with TB.A study of 100 patients hospitalized for TB in Madrid found presumed ocular TB in $24 \%$ of the $45 \mathrm{HIV}$-positive patients versus $13 \%$ in the $55 \mathrm{HIV}$-negative patients [34].Most patients have 
fewer than five lesions, but the number ranges from 1 to 60[5]. One or both eyes may be involved lesions are yellow, white, or gray. In active disease, overlying vitreous inflammation may be present. Inactive choroidal lesions appear as scars. The appearance of the choroiditis is not pathognomonic for TB, and similar lesions may be seen in sarcoidosis, syphilis and rarely metastatic disease. The choroidal tubercle may coalesce and produce a type of uveitis called serpiginous-like choroiditis[35].

Toxoplasmosis.0cular toxoplasmosis is the most common infectious cause of posterior uveitis in the United States. Toxoplasmosis is a worldwide infection that may cause a majority of posterior uveitis cases in highly endemic areas, such as Brazil, and France.

The characteristics funduscopic findings in ocular toxoplasmosis include a creamy yellow chorioretinal lesion adjacent to an old scar, and marked vitreous inflammation [I].Chorioretinitis in Aids patients is characterized by segmental panophthalmitis and areas of coagulative necrosis associated with tissue cysts and tachyzoites[36].Recent studies revealed a much higher incidence of ocular disease which is often severe, among infected immunocompetent people in South America than those in North America or Europe[[37].

Toxocariasis.0cular toxocariasis usually affects children and either is asymptomatic or causes unilateral decrease in vision. There are three types of ocular manifestations: (a) peripheral chorioretinal granuloma (50\% of cases)(b)posterior pole chorioretinal granuloma (25\%);and(c) diffuse panuveitis[38].In nearly all cases, only one eye is involved. Diagnosis may be difficult, especially in panuveitis cases.Becuase that infection is confined to the eye, serology id often negative and there usually no peripheral eosinophilia or oother signs of systemic parasitic infection. In addition, patients without ocular toxocariasis may have positive serologic test due to incidental or past exposure to Toxocara. A vitreous aspirate for evaluation of local Toxocara IgG compared with serum levels (Goldman-Witmer coefficient) may be helpful in diagnosis [39]. Lyme Disease. Uveitis rarely occurs in Lyme disease, but when it does the findings are protean. As with syphilis, ocular Lyme has been associated with anterior uveitis, intermediate uveitis, optic neuritis, neuroretinitis, retinal vasculitis, choroiditis, and panuveitis, Uveitis is usually seen in the late stage of Lyme disease [40,41]. Novel Infectious Agents of uveitis.These include: Leptospirosis, Brucellosis, Leprosy, Whipple's disease, Propinobacterium acne, Cat-scratch disease, West Nile virus, Candida spp , and Histoplasmosis.

\section{DIAGNOSIS OF UVEITIS}

Uveitis represents a diagnostic dilemma to ophthalmologist and infectious disease physicians. The diagnosis is nearly always presumptive and cannot be proved by pathology or culture. With rare exceptions, uvea cannot be biopsied without risking sight, so pathology of uvea is not available until the eye is lost. The aqueous and vitreous may be samples safely, but these samples rarely yield an infectious organism. Cytology of virectomy samples may be helpful to exclude malignancy (e.g. primary ocular lymphoma or metastatic cancer).Bacterial culture of the aqueous or vitreous are rarely positive.[1].In tuberculous uveitis, cultures of the vitreous and aqueous are nearly always negative. Similarly, viral cultures of aqueous and vitreous samples are rarely positive in herpetic uveitis, although PCR testing for herpesviruses is often positive [1].Gupta and colleagues reported that 10 of 17 patients (60\%) with presumed ocular TB had positive aqueous PCR assays for Mycobacterium tuberculosis, with clinical diagnosis based on a positive PPD or abnormal chest radiograph (or both)[42].

PCR has proven most helpful in herpetic uveitis cases, such as herpetic anterior uveitis and ARN. The aqueous or vitreous may be tested for HVS, VZV , or .CMV. Yamamoto and co-workers found that PCR tests of aqueous samples from 7 patients with recurrent iridocyclitis of suspected viral etiology were all positive for HVS(6 samples)VZV(1 sample), whereas samples from 17 control eyes were negative[43].In a study of 28 patients with ARN,PCR was positive in 27(96\%) for HSV, VZV or CMV[44].

Positive serologic tests, such as IgG antibodies to Toxoplasma, CMV, HVS, also be of little help, given the high prevalence of such antibodies in the general population. The one serologic test that always helpful and should be ordered on all patients with uveitis is the specific treponemal test for syphilis (e.g.,T.pallidum particle agglutination or FTA-abs).If this test is positive and eye findings are consistent with ocular syphilis, the patient should be treated for ocular syphilis.[45].The T.pallidum agglutination is preferred because the FTA-abs may be falsely positive in some patients, especially patients with rheumatologic conditions. A negative RPR does not exclude ocular syphilis because it is negative in greater than $50 \%$ of patients with eye disease with tertiary syphilis [46].

Radiologic studies of the eye or orbit sometimes are helpful in uveitis. Magnetic resonance imaging of the brain and orbit may be helpful in suspected cases of ocular-central nervous system lymphoma if brain lesions are found, although eye disease may precede central nervous system lesions by months. Fluorescein angiography of the eye may show retinal vascular patterns consistent with certain diseases, such as a viralinduced vasculitis in CMV .Indocyanine green angiography of the eye was found to be useful in detecting and following subclinical choroidal in eight patients with presumed ocular TB[47]. 


\section{THERAPY}

Herpetic anterior uveitis is treated primarily with topical corticosteroids. Oral acyclovir should be started as well, because long term prophylactic oral acylovir(400 $\mathrm{mg}$ twice daily) seems to be beneficial in preventing the recurrences of herpetic stromal keratitis and anterior uveitis[48,49].ARN due to HSV or VZV treated with high- dose intravenous acyclovir $(10 \mathrm{mg} / \mathrm{kg}$ every 8 hours with normal renal function) for 1 to 2 weeks followed by valacylovir or famiciclovir for 6 weeks to several months[22].Progressive outer retinal necrosis(PORN) has a dismal outcome despite therapy in many cases. Therapy with intravenous antiviral alone is rarely successful. Recent case reports have emphasized the importance of an aggressive medical approach however. There have been successes with repeated intravitreal injections with forscarnet and ganciclovir, in addition to prolonged combination IV therapy with these agents, and initiation of HAART in HIV-positive patients. In one patient with bilateral PORN, vision was lost in one eye, but an aggressive treatment led to visual recovery in the other eye [50].Ocular syphilis should be treated the same way as neurosyphilis with 10 to 14 days of intravenous penicillin (4 million $\mathrm{U}$ every 4 hours assuming a normal renal function).Systemic corticosteroids(e.g., prednisone, $80 \mathrm{mg}$ daily) should be started along with the antibiotic therapy, then tapered over days to weeks. Corticosteroids are given to decrease intraocular inflammation and prevent rebound inflammation from Jarisch- Herxheimer reaction [51].

Ocular TB should be treated with same medications and duration of therapy as TB meningitis. Although ethambutol is avoided because of potential ocular toxicity. Corticosteroids are helpful if there is sight threatening inflammation. Lyme uveitis should be treated the same as neuroborreliosis preferably with intravenous ceftriaxone[1].Treatment of ocular Toxoplasma, when peripheral lesions that do not threaten vision may not require treatment. Treatment for vision threatening lesions in adults includes sulfa drugs(e.g., sulfadiazine $1 \mathrm{~g}$ orally every 6 hours), pyrimethamine $(25 \mathrm{mg} /$ day orally) with folinic acid "rescue" $(5 \mathrm{mg} /$ day orally, and clindamycin(300 mg orally four times a day).Prednisone is often added in cases with severe vitreous inflammation and lesions threatening macula[52].Treatment of CMV includes drugs currently available that act to inhibit DNA polymerase are ganciclovir,(and valganciclovir), foscarnet, and cidofovir. Induction therapy includes 2 weeks or more of high dose drug. At times ganciclovir and foscarnet are given together to control relapsed or rapidly advancing disease(ocular or extra ocular). When retinitis is stable, patients are placed on lifelong maintenance therapy until there is evidence of immune recovery[53].

\section{VII.CONCLUSION}

Uveitis a sight threatening intraocular inflammation is prevalent worldwide. Recent medical advancement provides better understanding of pathogenesis and treatment of uveitis.

\section{REFERENCES}

[1]. Durand ML. Infectious Causes of Uveitis. In: Mandell, Douglas and Bennett's Principles and Practice of Infectious Diseases, $7^{\text {th }}$ Ed.Mandell GI.,Bennett JE,Dolin R(editors).Churchill Livingstone Elsevier,2010.

[2]. Subler ER,Ljoyd MJ,Choi D, et al.Incidence and prevalence of uveitis in veterans Affairs Medical Centers of Pacific North West. Am J Ophthalmol.2008;146:890-96.

[3]. Gritz Dc,Wong JG.Indidence and prevalence of uveitis in Northern California: The Northern California Epidemiology of Uveitis Study.Ophthalmology.2004;111:491-500

[4]. Ronday MJH,Stilma JS,Barbe RF,et al.Blindness from uveitis in a hospital population in Sierra Leone. $B r \quad J$ Ophthalmol.1994;78:690-95.

[5]. Helm CJ,Holland GN,0cular tuberculosis. Surv Ophthalmol.1993;38:229-56.

[6]. Jacob F, Reuland MS, Mackensen F,et al. Uveitis subtypes in a German interdisciplinary uveitis center-analysis of 1916 patients Rheumatol .Dec .15 .2008; epub ahead of print.

[7]. Shafik S,Foster CS.Definition,classification,etiology and epidemiology.In:Foster CS, Vitale AT,eds.Diagnosis and treatment of uveitis .Philadelphia: WB. Saunders, 2002; 17.

[8]. M.Ruiz-Cruz,Barrera CA,Terrazas YA,Teran RG'Proposed clinical use of definition for cyclomegalovirus-immune recovery retinitis".Clin Infect Dis.2014; View at Publisher -View at Google Scholar.

[9]. Rodriguez A,Calonge M.Pedoza-Seres M,et al.Referral patterns of uveitis in a tertiary eye care center.Arch Ophthalmol.1996;114:593-99

[10]. Karavellas MP,Plummer DJ,Macdonald JC,et al.Incidence of immune recovery vitritis in cytomegalovirus retinitis patients following institution of successful highly active antiretroviral therapy Infect Dis.1999;179(3),697-700.

[11]. Kupperman BD,Holland GN.Immune revovery uveitis.Am J Ophthalmol. 2000; 130 (1),103-6.

[12]. Modorati G,Miserocchi E,Brancato R.Immune recovery uveitis and human leukocyte antigen typing: a report on four patients. Eu J Ophthalmol.2005;15(5):607-9.

[13]. The Herpetic Eye Study Group. A controlled trial of oral acyclovir for iridocyclitis caused by herpes simplex virus. Arch Ophthalmol.1996; 114:1065-72.

[14]. Liesegang Y.0cular herpes simplex infection: Pathogenesis and current therapy. Myo Clin Proc.1988; 63:1092.

[15]. Pavan Langston D.Herpes simplex of the ocular anterior segment .In: Remington JS,Swartz MN.eds.Current Clinical Topics in Infectious Diseases 20 Cambridge. Mass:Blackwell Science;2000;296.

[16]. Kobashi Y,Hayasaka S,Shibuya Y,et al.Herpes simplex virus iridicyclitis unassociated with keratitis.Am Ophthalmol.1996;28:107-9.

[17]. Siverio CD Jr,Imati YD,Cunningham ET Jr.Diagnosis and management of herepetic anterior uveitis.Int Ophthalmol Clin.2002;12:43-8. 
[18]. Santos C.Herpes simplex uveitis.Bol ASoc Med P.R.2004;96:71-74.77-83.

[19]. Van der Lelii A,0oijman PM,Kiiletr A,et al.Anterior uveitis with sectroral iris atropathy in the absence of of keratitis.A distinct clinical entity among herepetic eye disease.0phthalmology.2000;107:1164-1670.

[20]. Urayamma A,Yamad N,Sasaki T,et al.Unilateral acute uveitis with retinal periarteritis and detachment.Jpn $J$ Clin Ophthalmol.1971;25:607-19.

[21]. Holland GN. Executive Committee of the American Uveitis Society. Standard diagnosis criteria for the acute retinal necrosis syndrome. Am J Ophthalmol. 1994: 117:663-67.

[22]. Lan CH,Missotten T,Slazmann J,et al.Acute retinal necrosis Features ,management, and outcomes.0phthalmology.2007;114:75662.

[23]. Tran THC,Stanescu D,Caspers-velu I,et al.Clinical characteristics of acute HSV-2 retinal necrosis.Am $J$ Ophthalmol.2004;137:872-79.

[24]. Paylay DA,Sternberg P,Davis J,et al.Decrease in the risk of bilateral acute retinal necrosis in acyclovir therapy. Am $J$ Ophthalmol.1991;112:250.

[25]. Chung H,Kim KH,Kim JG,et al.Retinal complications in patients with solid organ or bone marrow transplantation.Transplantation.2007;83:694-99.

[26]. Nakamoto BK,Dorothen EU,Biousse Y,et al.Progressive outer retinal necrosis presenting with isolated optic neuropathy.Neurology.2004;63:2423-25

[27]. Maves RC,Cachary ER,Young MA,et al.Secondary syphilis with ocular manifestation in older adults .Clin Infect Dis.2008;46:e142-e45.

[28]. Pleimes M,Hartschub W,Kutzner H,et al.Malignant syphilis with ocular involvement and organism-depleted lesions.Clin Infect Dis.2009;28:83-5.

[29]. Barilec GR,Flynn H.Syphilis exposure in patients with uveitis. Ophthalmology. 1997; 104:1605-9.

[30]. Becerra II,Ksiazeck SM,Savino PJ,et al.Syphilis uveitis in human immunodeficiency virus infected and noninfected patients.Ophthalmology.1989;96:1727-30.

[31]. Spoor TC,Ramocki JM,Nesi FA, et al. Ocular syphilis 1986.Prevalence of FTA-abs reactive and cerebrospinal fluid findings. J.Clin Neuroophthalmol.1987;7:191-95.

[32]. Donahue HC.Ophthamologic experience in a tuberculosis sanatorium. Am J Ophthamol.1967;64:742-48

[33]. Biswas J,Badrinath SS.0cular morbidity in patients with active systemic tuberculosis .Int Ophthalmol.1996;19:293-98.

[34]. Bouza F,Merino P,Munoz P,et al.0cular tuberculosis: A prospective study in a general hospital.Medicine.1997;76:53-61.

[35]. Gupta V,Gupta A,Ramby P,et al.Presumed tubercular serpiginous choroiditis:Clinical presentation and management.Ophthalmology.2003;110:1744-49.

[36]. Holland GN,0’Connor GR,et al.Toxoplasmosis .In:Pepose JS,Holland GN,Wilhelmus KR,eds.0cular infection and Immunity.St Louis:Mosby Yearbook;1996;1183-1223

[37]. Jones JI,Muccioli C,Belfort Jr R,et al. Recently acquired Toxoplasma gondii infection. Brazil Infect Dis.2006;12:582-87.

[38]. Stewart JM,Cubilan IT,Canningham FT Jr .Prevalence,clinical features, and causes of vision loss among cases of ocular toxocariasis.Retina.2005;25:1005-13.

[39]. De Visser I,Rothera A,de Boet JH,et al. Diagnosis of ocular toxoplasmosis by establishing intraocular antibody production.Am J Ophthalmol.2008;145:369-74.

[40]. Karma A,Seppala I,Mikkila H,et al.Diagnosis and clinical characteristics of ocular Lyme borreliosis.Am $J$ Ophthalmol.1995;119:127-35

[41]. Mikkila H0,Seppla R,Vilianen MK,et al.The expanding clinical spectrum of ocular Lyme borreliosis.Ophthalmology.2000;107:581-87.

[42]. Gupta V,Arora S,Gupta A,et al.Management of presumed intraocular tuberculosis: Possible role of the polymerase chain reaction. Acta Ophthalmol Scind.1998;76:679-82.

[43]. Yamamoto S,Pavan Langston D,Kinoshita S,et al.Detecting herpesvirus DNA in uveitis using the polymerase chain reaction. $B r J$ Ophthalmol.1996;80:465-468.

[44]. Ganatra JB,Chandley D,Santod C,et al.Viral causes of acute retinal necrosis syndrome.Am J 0phthalmol.2000;29:166-72.

[45]. MargoCE,Hamed IM.Ocular syphilis.Sury Ophthalmol.1992;37:203-20.

[46]. Murphy FT,George R,Kubota K,et al.The use of Western Blotting as the confirmatory test for syphilis in patients with rheumatic disease.J Rheumatol.1999;26:2448-53

[47]. Wollensherger TJ,Piquet R,Herbourt CP.Indocyanine green angiography features in tuberculous chorioretinitis.Am $J$ Ophthalmol.1999;127:390-53.

[48]. Herpetic Eye Disease Study Group.Oral acyclovir for herpes simplex virus eye disease: Effect on prevention of epithelial keratitis and stromal keratitis. Arch Ophthamol.2000;118:1030-36.

[49]. Rodriguez A,Power WJ,Neves RA,et al.Rucurrence rate of herpetic uveitis in patients on long -term oral acyclovir. Doc Ophthalmol.1995;90:331-40.

[50]. Yin Pd,Kurup SK,Fischer SH,et al. Progressive outer retinal necrosis in the era of highly active antiretroviral therapy: Successful management with intravitreal injection and monitoring with quantitative PCR.J Clin Virology.2007;38:254-59.

[51]. Fathilah J,Choo MM.The Jarisch-Hercheimer reaction in ocular syphilis. Med J Malaysia.2003;58:437-39.

[52]. Pearson PA,Piracha AR,Sen HA, et al.Atovaquone for the treatment of toxoplasma retinochoroiditis in immunocompetent patients.Ophthalmology.1999;106:148-53.

[53]. Jacobsen MA. Treatment of cytomegalovirus retinitis in patients with acquired immunodeficiency syndrome Engl $J$ Med.1997;337:105-14. 